\title{
Time-Varying System Identification via Maximum a posteriori Estimation and Its Application to Driver Steering Models
}

\author{
Tesheng Hsiao \\ Department of Electrical and Control Engineering \\ National Chiao Tung University, Hsinchu, Taiwan \\ tshsiao@cn.nctu.edu.tw
}

\begin{abstract}
Modern automotive technologies try to predict the driver's intention in order to control the vehicle effectively. However mathematical models describing the driver's steering behavior with sufficient accuracy are not available. The difficulties arise from the time-varying properties of the driver's behavior under rapidly changing traffic conditions. In this paper, a timevarying system identification method using maximum a posteriori estimation is proposed. An efficient iterative procedure is presented for maximizing the posterior probability of the parameters conditioning on observed data. Then it is applied to the experimental driving data, and the driver's time-varying steering models are identified and analyzed. The results indicate that the time-varying model reduces the output estimation errors significantly. Moreover, changes of driving strategies are observed from the identified models after drivers drive for a period of time.
\end{abstract}

\section{Introduction}

The recent development of advanced automotive technologies is aimed at enhancing driving safety when the driver is involved in the control loop. For example, CWS (collision warning system) issues alarms to draw the driver's attention whenever the vehicle is too close to the preceding one; ESP (electronic stability program) compares the driver's intention with the vehicle's response, correcting the vehicle's trajectory such that the driver maintains control of the vehicle. Because of the close interaction between the driver and the active or passive driving assistant systems, it is desirable to derive a mathematical model which describes and predicts the driver's behavior with sufficient accuracy.

The study of driver's steering model can be dated back to 1960's [1]. It has been shown that almost all manually controlled systems can be characterized by the "crossover model", i.e. the loop transfer function consisting of the driver and the vehicle behaves as an integrator around the gain crossover frequency [2]. As the advances in control theories, more structured driver models were proposed which took into account the driver's driving strategies, reaction time delay, and responses of neuromuscular systems [3][4].

Due to the complexity of human behavior, it is very difficult to derive the driver model from physical laws. On the other hand, system identification techniques allow researchers to establish models from simulated or experimental data. Chen, Pilutti, and Ulsoy used ARX and ARMAX models to represent the driver's steering behavior. System parameters and the range of model uncertainties were identified [5][6]; Kuge et al proposed an HMM-based framework to detect driving maneuvers. Then parameters of each sub-model were determined from driving data [7]. In these papers, the driving data were chopped by pieces and each piece of data was fit to an LTI system. Moreover the driving data came from driving simulators. The validity of the identified models depends on the accuracy of the simulator.

In order to investigate the driver's steering behavior under real traffic conditions instead of simulated environments, we will derive the driver model from the experimental data in this paper. It is observed from the data that drivers adjust their driving strategies frequently to accommodate the rapidly changing traffic. Therefore an LTI system can be an approximation of the driver's behavior for only a short period of time. As a result, the data length is too short to identify the time invariant parameters accurately.

In this paper the driver model will be represented by a time-varying system; hence techniques for identifying timevarying parameters are required. Various algorithms, such as adaptive filtering with forgetting factors [8], basis function expansion [9], and Bayesian inference methods [10], have been proposed for this purpose. Each method has its own strengths and weaknesses. Adaptive filtering is a natural extension of well-established adaptive algorithms; however it is suitable for slowly-varying systems. The basis function expansion method is computationally efficient, but its performance depends on the basis functions in use and the selection of the basis functions is not trivial. On the other hand, particle filtering and MCMC (Markov chain Monte Carlo) method treat all parameters as random variables and impose additional constraints in the form of conditional and prior probabilities. Then optimal estimates of the parameters are evaluated according to Bayesian inference principles. The problem formulation is straightforward; however the computation is intensive. To improve the efficiency while maintaining the structure of the Bayesian inference framework, we propose a maximum a posteriori (MAP) estimation method for time-varying parameters. MAP estimation for identifying time-varying AR systems has been shown to be computationally efficient [11]. In this paper it is extended to time- varying ARX (TVARX) systems which represent the driver's steering models. Then analysis based on the identified models is conducted.

This paper is organized as follows. Section II investigates the time-varying system identification method based on MAP estimation. It is applied to identify the driver's steer- 
ing model in Section III. Section IV concludes this paper.

\section{Identification of TVARX Systems II.1 Probabilistic Models}

TVARX systems can be expressed as follows.

$$
y(k)=-\sum_{i=1}^{n_{a}} a_{i}(k) y(k-i)+\sum_{j=0}^{n_{b}} b_{j}(k) u\left(k-j-n_{k}\right)+\varepsilon(k)
$$

where $u(k), y(k) \in \mathbb{R}$ are the input and output sequences respectively. $\varepsilon(k)$ is the process noise which is assumed to be a zero mean Gaussian distributed white noise with variance $\sigma_{\varepsilon}^{2}$ for all $k . a_{i}(k)$ and $b_{j}(k)$ for $i=1,2, \ldots, n_{a}$ and $j=0,1, \ldots, n_{b}$ are system parameters to be estimated. $n_{k}$ is the number of delay steps. $n_{a}, n_{b}$, and $n_{k}$ are positive integers and they are assumed to be known.

Given a set of input-output data $\left\{u(k), y(k) \mid k=-n_{m}, \ldots\right.$, $0,1, \ldots, N-1\}$, where $n_{m}=\max \left\{n_{a}, n_{b}+n_{k}\right\}$, we would like to find out the $\left(n_{a}+n_{b}+1\right) \times N$ parameters, $a_{i}(k)$ and $b_{j}(k)$, $i=1, \ldots, n_{a}, j=0, \ldots, n_{b}$, and $k=0, \ldots, N-1$ such that Eq.(1) is satisfied for all $k$. Apparently this is an under-determined problem and there exists infinitely many solutions. Therefore additional constraints must be imposed and an optimum criterion is required to evaluate the "best" solution. Bayesian inference achieves these goals in a natural way.

Under the Bayesian inference framework, all unknown parameters are regarded as random variables and additional constraints are imposed in terms of conditional probabilities and prior probabilities. Therefore $a_{i}(k)$ and $b_{j}(k)$ are assumed to be random variables for $i=1, \ldots, n_{a}, j=0, \ldots, n_{b}$ and $k=0, \ldots, N-1$. If the system's dynamics do not change violently, it is reasonable to assume that $a_{i}(k+1)$ stays in the neighborhood of $a_{i}(k)$ for all $i$ and $k$. Thus the following assumptions are made.

$$
\begin{aligned}
& a_{i}(k+1) \sim N\left(\cdot \mid a_{i}(k), \sigma_{a_{i}}^{2}\right), i=1, \ldots, n_{a}, k=0, \ldots, N-2 \\
& a_{i}(0) \sim N\left(\cdot \mid \mu_{a_{i}}, \sigma_{a_{i}}^{2}\right), i=1,2, \ldots, n_{a} \\
& a_{i}(k) \| a_{j}(l), i, j=1, \ldots, n_{a}, i \neq j ; k, l=0, \ldots, N-1
\end{aligned}
$$

where $N\left(\cdot \mid \mu, \sigma^{2}\right)$ denotes the Gaussian distribution with mean $\mu$ and variance $\sigma^{2} \cdot a_{i}(k) \| a_{j}(l)$ denotes that $a_{i}(k)$ and $a_{j}(l)$ are independent. $\mu_{a i}$ 's and $\sigma_{a i}$ 's in Eq.(2) and (3) are parameters of Gaussian distributions. Further discussion about these parameters will be presented shortly.

Similar assumptions are made for $b_{j}(k)$ 's as follows.

$$
\begin{aligned}
& b_{j}(k+1) \sim N\left(\cdot \mid b_{j}(k), \sigma_{b_{j}}^{2}\right), j=0, \ldots, n_{b}, k=0, \ldots, N-1 \\
& b_{j}(0) \sim N\left(\cdot \mid \mu_{b_{j}}, \sigma_{b_{j}}^{2}\right), j=0, \ldots, n_{b} \\
& b_{i}(k) \| b_{j}(l), \quad i, j=0, \ldots, n_{b}, i \neq j, k, l=0, \ldots, N-1
\end{aligned}
$$

We also assume that $a_{i}(k)$ and $b_{j}(l)$ are independent, i.e.

$$
a_{i}(k) \| b_{j}(l), i=1, \ldots, n_{a}, j=0, \ldots, n_{b}, k, l=0, \ldots, N-1
$$

Although additional constraints are imposed on $a_{i}(k)$ and $b_{j}(k)$ through Eq.(2)-(8), new parameters $\sigma_{a i}, \sigma_{b j}, \mu_{a i}$, and $\mu_{b j}$ for $i=1, \ldots, n_{a}$ and $j=0, \ldots, n_{b}$, are also introduced. Since there is no clue about the values or ranges of $\sigma_{a i}$ and $\sigma_{b}$, they can be regarded as random variables as well; thus a hierarchical structure of random variables is established. The probability distributions of $\sigma_{a i}$ and $\sigma_{b j}$, i.e. the prior distributions, reflect the designer's subjective belief in these variables and are somewhat arbitrary. In order to facilitate the subsequent derivations, conjugate prior probabilities of Gaussian distributions are assigned to $\sigma_{a i}{ }^{-2}$ and $\sigma_{b j}{ }^{-2}$. In other words, $\sigma_{a i}{ }^{-2}$ and $\sigma_{b j}^{-2}$ are assumed to be Gamma distributed.

$$
\begin{aligned}
& \sigma_{a_{i}}^{-2} \sim G a\left(\sigma_{a_{i}}^{-2} \mid \alpha_{a_{i}}, \beta_{a_{i}}^{-1}\right)=\frac{1}{\Gamma\left(\alpha_{a_{i}}\right) \beta_{a_{i}}^{-\alpha_{a_{i}}}}\left(\frac{1}{\sigma_{a_{i}}^{2}}\right)^{\alpha_{a_{i}}-1} \exp \left(\frac{-\beta_{a_{i}}}{\sigma_{a_{i}}^{2}}\right) \\
& \sigma_{b_{j}}^{-2} \sim G a\left(\sigma_{b_{j}}^{-2} \mid \alpha_{b_{j}}, \beta_{b_{j}}^{-1}\right)=\frac{1}{\Gamma\left(\alpha_{b_{j}}\right) \beta_{b_{j}}^{-\alpha_{b_{j}}}}\left(\frac{1}{\sigma_{b_{j}}^{2}}\right)^{\alpha_{b_{j}-1}} \exp \left(\frac{-\beta_{b_{j}}}{\sigma_{b_{j}}^{2}}\right)(10)
\end{aligned}
$$

Eq.(9) and (10) include hyper-parameters $\alpha_{a i}, \beta_{a i}, \alpha_{b i}$, and $\beta_{b j}$. It is possible to assume that these hyper-parameters are also random variables and their probability distributions can be assigned. This results in another hierarchy of random variables in the problem formulation. There is no restriction on the number of hierarch that can be established in a Bayesian inference framework; however there is no benefit to do that. Therefore these parameters will be assigned specific values which are chosen carefully by the designer.

Similarly, $\sigma_{\varepsilon}$ is also regarded as a random variable. Its prior probability distribution is assigned as follows.

$$
\sigma_{\varepsilon}^{-2} \sim G a\left(\sigma_{\varepsilon}^{-2} \mid \alpha_{\varepsilon}, \beta_{\varepsilon}^{-1}\right)
$$

where the hyper-parameters $\alpha_{\varepsilon}$ and $\beta_{\varepsilon}$ will be assigned specific values.

More assumptions about the relations among $\sigma_{a_{i}}^{-2}$, $\sigma_{b_{j}}^{-2}$ and $\sigma_{\varepsilon}^{-2}$ are considered. It is reasonable to assume that $\sigma_{a_{i}}^{-2}, \sigma_{b_{j}}^{-2}$ and $\sigma_{\varepsilon}^{-2}$ are mutually independent, i.e.

$$
\sigma_{a_{i}}^{-2}\left\|\sigma_{b_{j}}^{-2}\right\| \sigma_{\varepsilon}^{-2}, i=1, \ldots, n_{a}, j=0, \ldots, n_{b}
$$

\section{II.2 Posterior Probability}

To simplify the notation used in the subsequent derivations, the following vector notations are used.

$$
\begin{aligned}
& \mathbf{y}=[y(0), y(1), \ldots y(N-1)]^{\mathrm{T}} \in \mathbb{R}^{N} \\
& \mathbf{u}=\left[u\left(-n_{k}\right), \ldots u\left(N-1-n_{k}\right)\right]^{\mathrm{T}} \in \mathbb{R}^{N} \\
& \mathbf{a}_{i}=\left[a_{i}(0), a_{i}(1), \ldots, a_{i}(N-1)\right]^{\mathrm{T}} \in \mathbb{R}^{N}, \quad i=1, \ldots, n_{a} \\
& \mathbf{a}=\left[\mathbf{a}_{1}{ }^{\mathrm{T}}, \ldots, \mathbf{a}_{n a}{ }^{\mathrm{T}}\right]^{\mathrm{T}} \in \mathbb{R}^{N \cdot n a} \\
& \mathbf{b}_{j}=\left[b_{j}(0), b_{j}(1), \ldots, b_{j}(N-1)\right]^{\mathrm{T}} \in \mathbb{R}^{N}, \quad j=0, \ldots, n_{b} \\
& \mathbf{b}=\left[\mathbf{b}_{0}{ }^{\mathrm{T}}, \ldots, \mathbf{b}_{n b}{ }^{\mathrm{T}}\right]^{\mathrm{T}} \in \mathbb{R}^{N \cdot(n b+1)} \\
& \boldsymbol{\sigma}_{a}^{-2}=\left[\begin{array}{llll}
\sigma_{a_{1}}^{-2} & \sigma_{a_{2}}^{-2} & \cdots & \sigma_{a_{n_{a}}}^{-2}
\end{array}\right]^{T} \in \mathbb{R}^{n_{a}} \\
& \boldsymbol{\sigma}_{b}^{-2}=\left[\begin{array}{llll}
\sigma_{b_{0}}^{-2} & \sigma_{b_{1}}^{-2} & \cdots & \sigma_{b_{n_{b}}}^{-2}
\end{array}\right]^{T} \in \mathbb{R}^{n_{b}+1}
\end{aligned}
$$

The posterior probability $p(\mathbf{a}, \mathbf{b} \mid \mathbf{y})$ is the marginal probability of $p\left(\mathbf{a}, \mathbf{b}, \boldsymbol{\sigma}_{a}^{-2}, \boldsymbol{\sigma}_{b}^{-2}, \sigma_{\varepsilon}^{-2} \mid \mathbf{y}\right)$, i.e. 
$p(\mathbf{a}, \mathbf{b} \mid \mathbf{y})=\iiint p\left(\mathbf{a}, \mathbf{b}, \boldsymbol{\sigma}_{a}^{-2}, \boldsymbol{\sigma}_{b}^{-2}, \sigma_{\varepsilon}^{-2} \mid \mathbf{y}\right) d\left(\boldsymbol{\sigma}_{a}^{-2}\right) d\left(\boldsymbol{\sigma}_{b}^{-2}\right) d\left(\sigma_{\varepsilon}^{-2}\right)$

From Baye's theorem and the indepence assumptions Eq.(8) and (12), we have

$p\left(\mathbf{a}, \mathbf{b}, \boldsymbol{\sigma}_{a}^{-2}, \boldsymbol{\sigma}_{b}^{-2}, \sigma_{\varepsilon}^{-2} \mid \mathbf{y}\right)$

$\propto p\left(\mathbf{y} \mid \mathbf{a}, \mathbf{b}, \boldsymbol{\sigma}_{a}^{-2}, \boldsymbol{\sigma}_{b}^{-2}, \sigma_{\varepsilon}^{-2}\right) p\left(\mathbf{a} \mid \boldsymbol{\sigma}_{a}^{-2}\right) p\left(\mathbf{b} \mid \boldsymbol{\sigma}_{b}^{-2}\right) p\left(\boldsymbol{\sigma}_{a}^{-2}\right) p\left(\boldsymbol{\sigma}_{b}^{-2}\right) p\left(\sigma_{\varepsilon}^{-2}\right)$

Since $\varepsilon(k)$ is assumed to be Gaussian distributed, we have $p\left(\mathbf{y} \mid \mathbf{a}, \mathbf{b}, \boldsymbol{\sigma}_{a_{i}}^{-2}, \boldsymbol{\sigma}_{b_{i}}^{-2}, \sigma_{\varepsilon}^{-2}\right)$

$\propto\left(\sigma_{\varepsilon}^{-2}\right)^{\frac{N}{2}} \exp \left(\frac{-1}{2 \sigma_{\varepsilon}^{2}} \sum_{k=0}^{N-1}\left(y(k)+\sum_{i=1}^{n_{s}} a_{i}(k) y(k-i)-\sum_{j=0}^{n_{b}} b_{j}(k) u\left(k-j-n_{k}\right)\right)^{2}\right)$

On the other hand, from Eq.(2)-(4) and (9), we get $p\left(\mathbf{a} \mid \boldsymbol{\sigma}_{a}^{-2}\right)$

$=\prod_{i=1}^{n_{a}} p\left(\mathbf{a}_{i} \mid \sigma_{a_{i}}^{-2}\right)$

$=\prod_{i=1}^{n_{a}}\left(\prod_{k=1}^{N-1} p\left(a_{i}(k) \mid a_{i}(k-1), \sigma_{a_{i}}^{-2}\right) p\left(a_{i}(0) \mid \sigma_{a_{i}}^{-2}\right)\right)$

$\propto \prod_{i=1}^{n_{a}}\left(\sigma_{a_{i}}^{-2}\right)^{\frac{N}{2}} \exp \left(\frac{-1}{2 \sigma_{a_{i}}^{2}}\left[\sum_{k=1}^{N-1}\left(a_{i}(k)-a_{i}(k-1)\right)^{2}+\left(a_{i}(0)-\mu_{a_{i}}\right)^{2}\right]\right)$

Similarly,

$p\left(\mathbf{b} \mid \boldsymbol{\sigma}_{b}^{-2}\right)$

$\propto \prod_{j=0}^{n_{b}}\left(\sigma_{b_{j}}^{-2}\right)^{\frac{N}{2}} \exp \left(\frac{-1}{2 \sigma_{b_{j}}^{2}}\left[\sum_{k=1}^{N-1}\left(b_{j}(k)-b_{j}(k-1)\right)^{2}+\left(b_{j}(0)-\mu_{b_{j}}\right)^{2}\right]\right)$

Substituting $p\left(\mathbf{a} \mid \boldsymbol{\sigma}_{a}^{-2}\right), p\left(\mathbf{b} \mid \boldsymbol{\sigma}_{b}^{-2}\right)$ and Eq.(9) and (10) into Eq. (13) and computing the integration by repeatly applying integration by parts, we obtain the following posterior probability $p(\mathbf{a}, \mathbf{b} \mid \mathbf{y})$.

$$
p(\mathbf{a}, \mathbf{b} \mid \mathbf{y}) \propto g(\mathbf{a}, \mathbf{b} \mid \mathbf{y}) \prod_{i=1}^{n_{a}} f_{a_{i}}\left(\mathbf{a}_{i}\right) \prod_{j=0}^{n_{b}} f_{b_{j}}\left(\mathbf{b}_{j}\right)
$$

where

$$
\begin{aligned}
& g(\mathbf{a}, \mathbf{b} \mid \mathbf{y}) \\
& =\left[\frac{1}{2} \sum_{k=0}^{N-1}\left(y(k)+\sum_{i=1}^{n_{a}} a_{i}(k) y(k-i)-\sum_{j=0}^{n_{b}} b_{j}(k) u\left(k-j-n_{k}\right)\right)^{2}+\beta_{\varepsilon}\right]^{-p} \\
& f_{a_{i}}\left(\mathbf{a}_{i}\right)=\left[\frac{1}{2} \sum_{k=1}^{N-1}\left(a_{i}(k)-a_{i}(k-1)\right)^{2}+\frac{1}{2}\left(a_{i}(0)-\mu_{a_{i}}\right)^{2}+\beta_{a_{i}}\right]^{-p} \\
& f_{b_{j}}\left(\mathbf{b}_{j}\right)=\left[\frac{1}{2} \sum_{k=1}^{N-1}\left(b_{j}(k)-b_{j}(k-1)\right)^{2}+\frac{1}{2}\left(b_{j}(0)-\mu_{b_{j}}\right)^{2}+\beta_{b_{j}}\right]^{-p}
\end{aligned}
$$

For simplicity, the hyper-parameters $\alpha_{a i}, \alpha_{b j}$ and $\alpha_{\varepsilon}$, are chosen such that $p=\frac{N}{2}+\alpha_{a_{i}}=\frac{N}{2}+\alpha_{b_{j}}=\frac{N}{2}+\alpha_{\varepsilon} \in \mathbb{N}$ for all $i=1, \ldots, n_{a}$ and $j=0, \ldots, n_{b}$.

\section{II.3 Maximum a posteriori Estimation}

Let $\left(\hat{\mathbf{a}}_{M A P}, \hat{\mathbf{b}}_{M A P}\right)$ be the maximizer of the posterior probability $p(\mathbf{a}, \mathbf{b} \mid \mathbf{y})$, i.e. $\left(\hat{\mathbf{a}}_{M A P}, \hat{\mathbf{b}}_{M A P}\right)=\arg \max _{\mathbf{a}, \mathbf{b}} p(\mathbf{a}, \mathbf{b} \mid \mathbf{y})$. $\left(\hat{\mathbf{a}}_{M A P}, \hat{\mathbf{b}}_{M A P}\right)$ is called the maximum a posteriori (MAP) estimate of the unknown parameters a and b. MAP is a reasonable optimum criterion for evaluating the best estimate of $\mathbf{a}$ and $\mathbf{b}$ becuase $\left(\hat{\mathbf{a}}_{M A P}, \hat{\mathbf{b}}_{M A P}\right)$ is the most likely value of $\mathbf{a}$ and $\mathbf{b}$, conditioning on observered data $\mathbf{y}$.

Since $\left(\hat{\mathbf{a}}_{M A P}, \hat{\mathbf{b}}_{M A P}\right)$ maximizes $p(\mathbf{a}, \mathbf{b} \mid \mathbf{y})$, the first derivative of $p(\mathbf{a}, \mathbf{b} \mid \mathrm{y})$ w.r.t. $\mathbf{a}$ and $\mathbf{b}$ vanishes at $\left(\hat{\mathbf{a}}_{M A P}, \hat{\mathbf{b}}_{M A P}\right)$, i.e.

$$
\begin{aligned}
& \left.\frac{\partial p(\mathbf{a}, \mathbf{b} \mid \mathbf{y})}{\partial a_{i}(k)}\right|_{\substack{\mathbf{a}=\hat{\mathbf{a}}_{M A P} \\
\mathbf{b}=\mathbf{b}_{M A P}}}=0, i=1,2, \ldots, n_{a}, k=0,1, \ldots, N-1 \\
& \left.\frac{\partial p(\mathbf{a}, \mathbf{b} \mid \mathbf{y})}{\partial b_{j}(k)}\right|_{\substack{\mathbf{a}=\hat{\mathbf{a}}_{M A P} \\
\mathbf{b}=\hat{\mathbf{b}}_{M A P}}}=0, j=0,1, \ldots, n_{b}, k=0,1, \ldots, N-1
\end{aligned}
$$

Eq.(15) and (16) consist of $\left(n_{a}+n_{b}+1\right) \cdot N$ nonlinear equations. They must be solved simutaneously in order to get the closed-form solution of $\hat{\mathbf{a}}_{M A P}$ and $\hat{\mathbf{b}}_{M A P}$. Unfortunately, Eq.(15) and (16) are too complicated to be solved analytically. Therefore, an iterative procedure is proposed that manipulates one variable at a time and let all the others hold their values from the previous iteration. Suppose that $\hat{\mathbf{a}}^{(t)} \in \mathbb{R}^{n_{a} \cdot N}$ and $\hat{\mathbf{b}}^{(t)} \in \mathbb{R}^{\left(n_{b}+1\right) \cdot N}$ are obtained as an approximation of $\hat{\mathbf{a}}_{M A P}$ and $\hat{\mathbf{b}}_{M A P}$ respectively at the $t$-th iteration. Then the elements of $\hat{\mathbf{a}}^{(t)}$ and $\hat{\mathbf{b}}^{(t)}$ are updated one by one into $\hat{\mathbf{a}}^{(t+1)}$ and $\hat{\mathbf{b}}^{(t+1)}$. For each update, only one variable in one equation of Eq.(15) and (16) needs taking care of; hence the complexity of the problem is reduced. The procedure goes on iteratively in a way that drives $\hat{\mathbf{a}}^{(t)}$ and $\hat{\mathbf{b}}^{(t)}$ to the local maximum of $p(\mathbf{a}, \mathbf{b} \mid \mathbf{y})$ as $t$ approaches infinity.

If at the $t$-th iteration, $a_{i}(k)$ is going to be updated for some $i$ and $k$, Eq.(14) is rewritten as a function of that single variable $a_{i}(k)$ while the other parameters are set to be the values at the $(t-1)$-th iteration. Hence we have

$$
\begin{aligned}
& f_{a_{i}}\left(\mathbf{a}_{i}\right) \\
= & f_{a, i, k}\left(a_{i}(k) \mid a_{i}(0), \ldots, a_{i}(k-1), a_{i}(k+1), \ldots, a_{i}(N-1)\right) \\
\triangleq & \left\{\delta(k) \eta_{a i}^{2}(k)\left[1+\left(\frac{a_{i}(k)-c_{a i}(k)}{\eta_{a i}(k)}\right)^{2}\right]\right\}^{-p}
\end{aligned}
$$

where $\delta(N-1)=0.5$ and $\delta(k)=1$ for $k=0,1, \ldots, N-2$,

$$
c_{a i}(k)= \begin{cases}a_{i}(N-2), & k=N-1 \\ \frac{1}{2}\left(a_{i}(k-1)+a_{i}(k+1)\right), & 0<k<N-1 \\ \frac{1}{2}\left(a_{i}(1)+\mu_{a_{i}}\right) & k=0\end{cases}
$$

and 


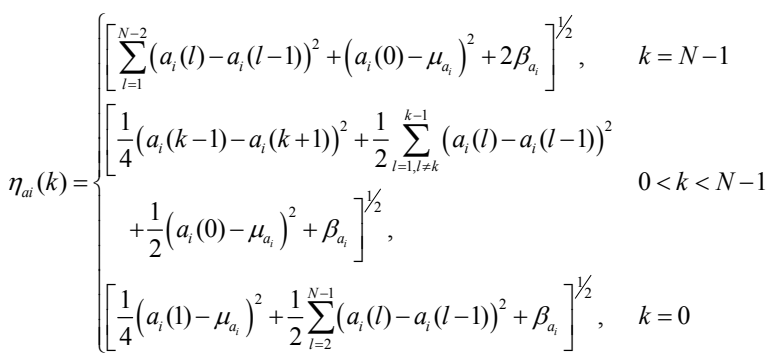

On the other hand,

$$
\begin{aligned}
& g(\mathbf{a}, \mathbf{b} \mid \mathbf{y}) \\
& =g_{a, i, k}\left(a_{i}(k) \mid \mathbf{a}_{-i, k}\right)
\end{aligned}
$$

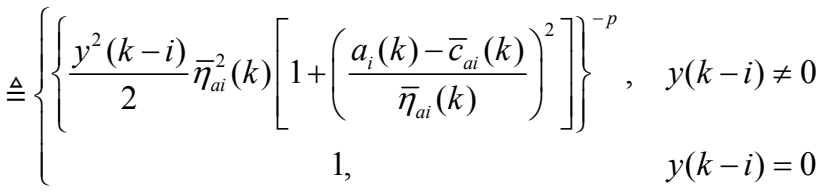

where $\mathbf{a}_{-i, k}$ denotes a $\mathbb{R}^{N \cdot n a-1}$ vector consisting of all parameters in a except $a_{i}(k)$. Besides,

$$
\begin{aligned}
\bar{c}_{a i}(k)=\frac{-1}{y(k-i)}\left(y(k)+\sum_{l=1, l \neq i}^{n_{a}} a_{l}(k) y(k-l)-\sum_{j=0}^{n_{b}} b_{j}(k) u\left(k-j-n_{k}\right)\right) \\
\bar{\eta}_{a i}(k)=\frac{1}{|y(k-i)|}\left[\sum _ { m = 0 , m \neq k } ^ { N - 1 } \left(y(m)+\sum_{l=1}^{n_{a}} a_{l}(m) y(m-l)\right.\right. \\
\left.\left.-\sum_{j=0}^{n_{b}} b_{j}(m) y\left(m-j-n_{k}\right)\right)^{2}+2 \beta_{\varepsilon}\right]^{1 / 2}
\end{aligned}
$$

Note that if $y(k-i)=0, \quad \bar{c}_{a i}(k)$ and $\bar{\eta}_{a i}(k)$ are not well-defined. But in this case, $g(\mathbf{a}, \mathbf{b} \mid \mathbf{y})$ is independent of $a_{i}(k)$ and becomes a constant w.r.t. $a_{i}(k)$. In other words,

$p(\mathbf{a}, \mathbf{b} \mid \mathbf{y}) \propto f_{i, k}\left(a_{i}(k) \mid a_{i}(0), \ldots, a_{i}(k-1), a_{i}(k+1), \ldots, a_{i}(N-1)\right)$

Thus define $g_{a, i, k}\left(a_{\mathrm{i}}(k) \mid \mathbf{a}_{-i, k}\right)=1$ whenever $y(k-i)=0$.

According to the definition of $f_{a, i, k}$ and $g_{a, i, k}$, Eq.(15) becomes

$\frac{d}{d\left(a_{i}(k)\right)}\left\{\left[1+\left(\frac{a_{i}(k)-c_{a i}(k)}{\eta_{a i}(k)}\right)^{2}\right]\left[1+\left(\frac{a_{i}(k)-\bar{c}_{a i}(k)}{\bar{\eta}_{a i}(k)}\right)^{2}\right]\right\}^{-p}=0$

By straightforward calculation, Eq.(21) is equivalent to

$$
\begin{aligned}
& a^{3}-\frac{3}{2}\left(c_{a}+\bar{c}_{a}\right) a^{2}+\frac{1}{2}\left(c_{a}^{2}+\bar{c}_{a}^{2}+4 c_{a} \bar{c}_{a}+\eta_{a}^{2}+\bar{\eta}_{a}^{2}\right) a \\
& -\frac{1}{2}\left[c_{a}\left(\bar{c}_{a}^{2}+\bar{\eta}_{a}^{2}\right)+\bar{c}_{a}\left(c_{a}^{2}+\eta_{a}^{2}\right)\right]=0
\end{aligned}
$$

The subscript $i$ and time index $k$ of all variables are dropped for a simple expression. Note that the left hand side of Eq.(22) is a third order polynomial. At least one of its three roots must be real and these roots can be found analytically without applying numerical methods; thus the calculation of the roots can be accomplished efficiently. The root that achieves the maximum value of $p(\mathbf{a}, \mathbf{b} \mid \mathbf{y})$ is the estimate of $a_{\mathrm{i}}(k)$ at the current iteration.

On the other hand, if $b_{j}(k)$ is going to be updated, Eq.(14) is rewritten as a function of a single variable $b_{j}(k)$ in a similar way, i.e. $p(\mathbf{a}, \mathbf{b} \mid \mathbf{y}) \propto\left\{\delta(k)\left[1+\left(\frac{b_{j}(k)-c_{b j}(k)}{\eta_{b j}(k)}\right)^{2}\right]\left[1+\left(\frac{b_{j}(k)-\bar{c}_{b j}(k)}{\bar{\eta}_{b j}(k)}\right)^{2}\right]\right\}^{-p}$

where the definitions of $c_{b j}(k), \eta_{b j}(k), \bar{c}_{b j}(k)$, and $\bar{\eta}_{b j}(k)$ are similar to Eq.(17)-(20).

Maximizing $p(\mathbf{a}, \mathbf{b} \mid \mathbf{y})$ w.r.t. $b_{j}(k)$ ends up with solving a third order polynomial which replaces $c_{a}, \eta_{a}, \bar{c}_{a}$, and $\bar{\eta}_{a}$ in Eq.(22) with $c_{b}, \eta_{b}, \bar{c}_{b}$, and $\bar{\eta}_{b}$ respectively. Then the real root that achieves the maximum value of $p(\mathbf{a}, \mathbf{b} \mid \mathbf{y})$ is selected as the estimate of $b_{j}(k)$. The detailed derivation is skipped because it follows exactly the same procedures presented in the previous paragraphs.

In summary, we propose the following algorithm to estimate the time-varying parameters of the ARX system.

Algorithm I: MAP estimation of TVARX system parameters Given hyper-parameters $\beta_{\varepsilon}, \beta_{a i}, \beta_{b j}, \mu_{a i}$ and $\mu_{b j}$, for $i=1, \ldots, n_{a}$, and $j=0, \ldots, n_{b}$. Let $\hat{\mathbf{a}}^{(t)}$ and $\hat{\mathbf{b}}^{(t)}$ be the estimate of $\mathbf{a}$ and $\mathbf{b}$ respectively at the $t$-th iteration.

At the $(t+1)$-th iteration

For $k=0,1, \ldots, N-1$

For $i=1,2, \ldots, n_{a} \quad\{$

1. Calculate $c_{a i}(k)$ and $\eta_{a i}(k)$ (Eq.(17)-(18))

2. If $y(k-i)=0$ $\hat{a}_{i}^{(t+1)}(k)=c_{a i}(k)$; exist the For loop

3. Calculate $\bar{c}_{a i}(k)$ and $\bar{\eta}_{a i}(k) \quad$ (Eq. $\left.\quad-(20)\right)$

4. Find $r_{m}, 1 \leq m \leq 3$, the real solution(s) to Eq.(22)

5. $\left.\hat{a}_{i}^{(t+1)}(k)=\arg \max _{1 \leq m \leq 3} p\left(r_{m}, \hat{\mathbf{a}}_{-i, k}^{(t)}, \hat{\mathbf{b}}^{(t)} \mid \mathbf{y}\right) \quad\right\}$

For $j=0,1, \ldots, n_{b} \quad\{$

6. Calculate $c_{b j}(k)$ and $\eta_{b j}(k)$

7. If $y(k-i)=0$

$\hat{b}_{j}^{(t+1)}(k)=c_{b j}(k)$; exist the For loop

8. Calculate $\bar{c}_{b j}(k)$ and $\bar{\eta}_{b j}(k)$

9. Find $r_{m}, 1 \leq m \leq 3$, the real solution(s) to polynomial which is the counterpart of Eq.(22)

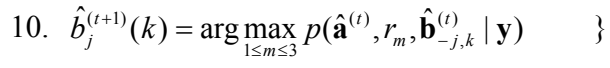

\}

\section{II.4 An Illustrative Example}

Consider the following TVARX system

$$
\begin{aligned}
y(k)= & -a_{1}(k) y(k-1)-a_{2}(k) y(k-2)+b_{0}(k) u(k-1) \\
& +b_{1}(k) u(k-2)+b_{3}(k) u(k-3)+\varepsilon(k)
\end{aligned}
$$

Assume that $\sigma_{\varepsilon}=0.05$. Apply Algorithm I to identify the time-varying parameters $a_{1}(k), a_{2}(k), b_{0}(k), b_{1}(k)$, and $b_{2}(k)$ for $\mathrm{k}=0.1, \ldots, 99$. After 2000 iterations, the parameters converges and the identified parameters are shown in Figure 1. 

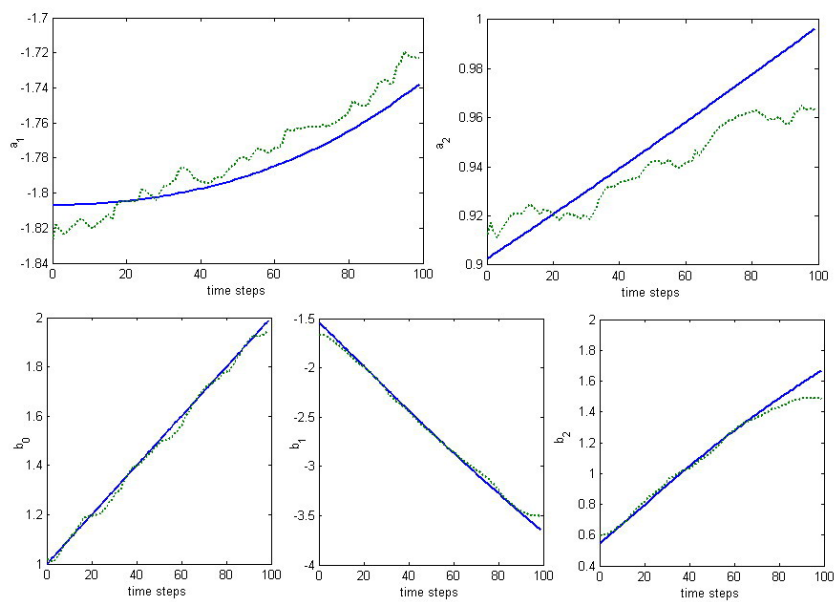

Figure 1: Time-varying parameters and the estimated values. Upper row (from left to right): $a_{1}$ and $a_{2}$. Lower row (from left to right): $b_{0}, b_{1}$ and $b 2$. Real line (-): true parameters. Dotted line (.): estimated parameters.
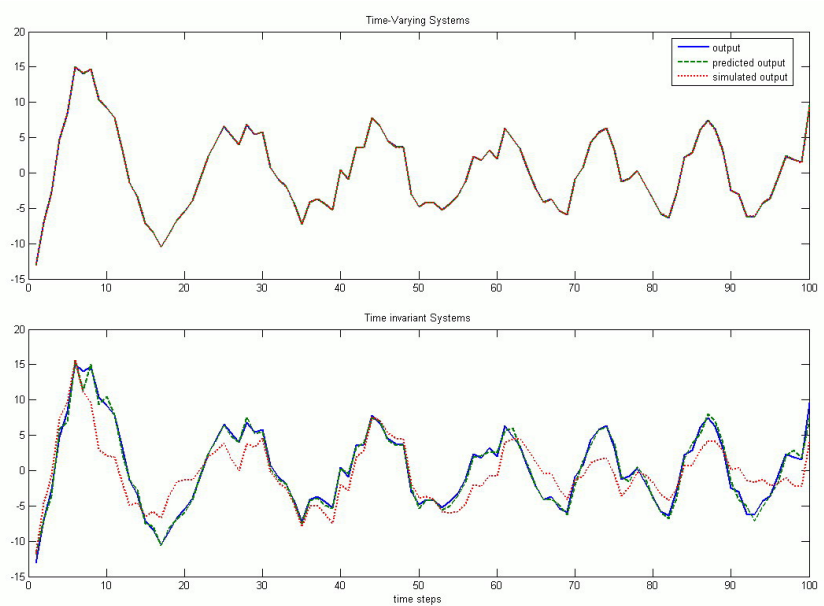

Figure 2: Upper: parameters are time varying. Lower: parameters are time-invariant. Real line (-): measured output. Dash line (--): predicted output. Dotted line (.): simulated output.

It can be seen that the proposed method can effectively identify the time-varying parameters. If the same input and output data is fit to an LTI system, the resulting time-invariant parameters are $\hat{a}_{1}=-1.7264, \hat{a}_{2}=0.8514$, $\hat{b}_{0}=1.3009, \hat{b}_{1}=-2.3461$, and $\hat{b}_{2}=0.7322$. Figure 2 illustrates the estimated outputs for time-varying and time-invariant parameters. Note that the predicted output is defined as

$$
\begin{aligned}
\hat{y}_{p}(k) & =-\hat{a}_{1}(k) y(k-1)-\hat{a}_{2}(k) y(k-2)+\hat{b}_{0}(k) u(k-1) \\
& +\hat{b}_{1}(k) u(k-2)+\hat{b}_{3}(k) u(k-3)
\end{aligned}
$$

whereas the simulated output is defined as

$$
\begin{aligned}
\hat{y}_{s}(k) & =-\hat{a}_{1}(k) \hat{y}_{s}(k-1)-\hat{a}_{2}(k) \hat{y}_{s}(k-2)+\hat{b}_{0}(k) u(k-1) \\
& +\hat{b}_{1}(k) u(k-2)+\hat{b}_{3}(k) u(k-3)
\end{aligned}
$$

It is obvious from this figure that time-varying system representation is superior to the time-invariant system representation in terms of simulated output errors.

\section{Driver Steering Models}

III.1 Driving Data under Real Traffic Conditions

The closed-loop vehicle lane-keeping control system is illustrated in Figure 3. The vehicle changes the lateral position of its center of gravity $y_{\text {cg }}$, as well as the yaw angle $\varphi$ whenever a steering command $\delta$ is given. The driver perceives the changes of the vehicle's motion by observing the vehicle's lateral position at some look-ahead distance $y_{\mathrm{a}}$. The difference between $y_{\mathrm{a}}$ and the desired lateral potion $y_{\mathrm{d}}$ is sensed by the driver and then the steering command $\delta$ is adjusted accordingly.

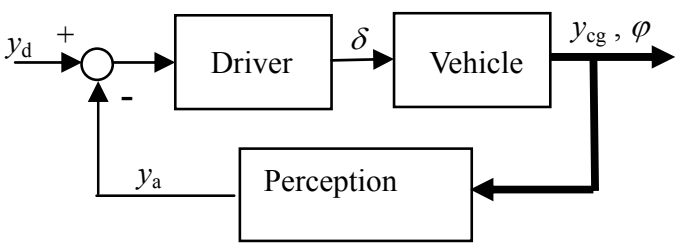

Figure 3: The block diagram of the vehicle lane-keeping control system consisting of the vehicle and the driver.

For the lane-keeping control purpose, $y_{\mathrm{d}}$ is set to be zero, i.e. the driver maintains the vehicle moving along the road centerline. In this case the input to the driver steering model is the lateral position at some look-ahead distance, $y_{\mathrm{a}}$, while the output is the steering angle $\delta$. The steering angle can be measured by the potentiometer mounted on the steering column. To measure the lateral position, the instrumented vehicle is equipped with a vision system which calculates from the images the lateral position at the gazing point ahead of the vehicle. The sampling time of the measured data is $75 \mathrm{~ms}$.

In the experiments, subjects were asked to drive the instrumented vehicle on highways for about one hour. The longitudinal speed, steering angle, and lateral position of the vehicle were recorded in the on-board storage devices. These data are analyzed in this section to identify the driver models.

Since the driver's response is slower than $1 \mathrm{~Hz}$ [4], the steering angle and the lateral position are pre-filtered by a low-pass filter with cutoff frequency at $1 \mathrm{~Hz}$ to remove the measurement noise. DC bias is also removed from the input and output data such that the vehicle has no lateral offset in steady state.

Motivated by the discussion in [3] and [4], we select the model order to be $\left(n_{a}, n_{b}, n_{k}\right)=(4,4,2)$. Two subjects are selected randomly from the database and their driving data are divided into 2-minute segments. Then Algorithm I is applied to these data segments to identify the time-varying parameters of the driver's steering model. Analysis of the identified models is conducted in the remainder of this section. 
Firstly we show that the time-varying model reduces the simulated output errors significantly. The driving data are fit to both the TVARX and ARX models. Then the predicted and simulated output errors are demonstrated in Figure 4. It can be seen that under real traffic conditions, a single LTI system is not able to characterize the driver's steering behavior for a 2-minute period of time. However the timevarying model reproduces the data faithfully.
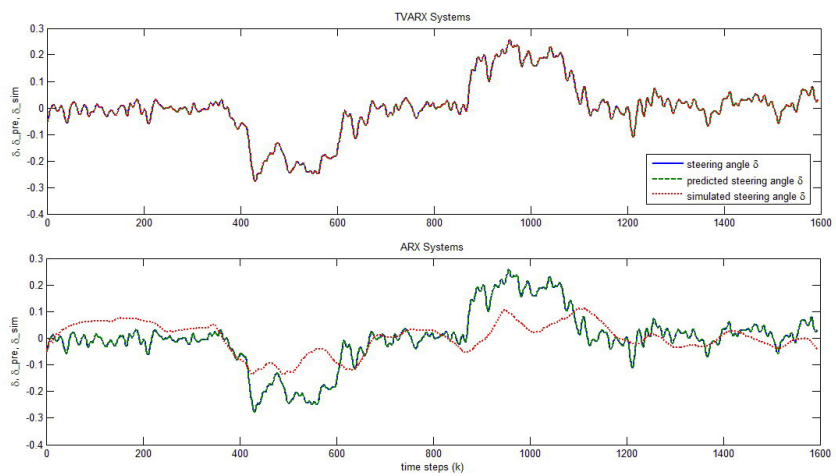

Figure 4: Upper: TVARX systems. Lower: ARX systems. Real line (-): steering angle. Dash line (--): predicted steering angle. Dotted line (.): simulated steering angle.

Figure 5 illustrates the instantaneous zero locations at each time step. The left column and the right column represent Subject 1 and Subject 2 respectively. The upper row is based on the data at the beginning of the test drive while the lower row is based on the data after 29'58" for Subject 1, and 61'28" for Subject 2. It is observed that the zeros of both subjects' models spread over wider areas after driving for half an hour or longer. The zero locations can be regarded as the driving strategies of individual drivers [12]. The change of driving strategies after driving for a period of time may be an indication of fatigue. However more research efforts are required to investigate the correlations between the changes of zero patterns and other factors.
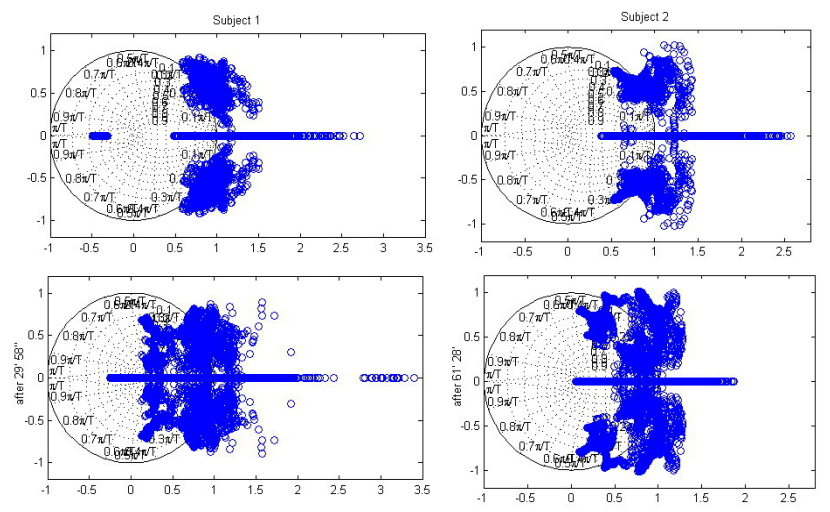

Figure 5: Instantaneous zero locations. Left column: Subject 1. Right column: Subject 2. Upper row: at the beginning of the test drive. Lower row: after $29 \mathrm{~min}$ and $58 \mathrm{sec}$ for Subject 1 , and $61 \mathrm{~min}$ and $28 \mathrm{sec}$ for Subject 2.

\section{Conclusion}

In this paper, a system identification method for TVARX models was proposed. An illustrative example demonstrated that the proposed method is able to estimate the time-varying parameters reasonably well. The computation is also efficient. Then the proposed method is applied to the driving data collected under real traffic conditions to identify time-varying driver steering models. It was shown that time-varying models reduce the simulated output errors significantly. Moreover, changes of zero patterns were observed at different time segments for both randomly selected subjects. Further investigations are required to establish the correlation between the change of zero patterns and other factors such as fatigue and/or specific traffic conditions.

\section{References}

[1] J. G.. Wohl, "Man-machine Steering Dynamics," Human Factors, vol. 3, no. 4, pp. 222-228, 1961

[2] D. T. McRuer and E. S. Kredel, "Mathematical Models of Human Pilot Behavior," AGARD-AG-188, 1974

[3] R. W. Allen, "Stability and Performance Analysis of Automobile Driver Steering Control," SAE paper 820303, 1982

[4] R. A. Hess and A. Modjtahedzadeh, "A Control Theoretic Model of Driver Steering Behavior," IEEE Control Systems Magazine, vol.10, no. 5, pp. 3-8, 1990

[5] L. K. Chen and A. G. Ulsoy, "Identification of a Driver Steering Model and Model Uncertainty, from Driving Simulator Data," ASME Journal of Dynamic Systems, Measurement, and Control, vol. 123, pp. 623-629, 2001

[6] T. Pilutti and A.G. Ulsoy, "Identification of Driver State for Lane-keeping Tasks," IEEE Transactions on Systems, Man and Cybernetics-PART A: Systems and Humans, vol. 29, no. 5, pp. 486-502, 1999

[7] N. Kuge et al, "A Driver Behavior Recognition Method Based on a Driver Model Framework," SAE paper 2000-01-0349, 1998

[8] J. C. M. Bermudez and N. I. Bershad, "Transient and Tracking Performance Analysis of the Quantized LMS Algorithm for Time-Varying System Identification," IEEE Transactions on Signal Processing, vol. 44, pp. 1990-1997, 1996

[9] M. K. Tsatsanis and G. B. Giannakis, "Time-Varying System Identification and Model Validation Using Wavelets," IEEE Transactions on Signal Processing, vol. 41, pp. 3512-3523, 1993

[10] S. Godsill and T. Clapp, "Improvement Strategies for Monte Carlo Particle Filters," in Sequnetial Monte Carlo Methods in Practice, A. Doucet, N. d. Freitas, and N. Gordon, Eds.: Springer, 2001, pp. 139-158

[11] T. Hsiao, "Identification of Time-Varying Autoregressive Systems Using Maximum a posteriori Estimation," to appear in IEEE transactions on Signal Processing

[12] S. Horiuchi and N. Yuhara, "An Analytical Approach to the Prediction of Handling Qualities of Vehicles with Advanced Steering Control System Using Multi-Input Driver Model," Transaction on ASME, Vol. 122, pp490-497, 2000 